\title{
Trauma ocular ocupacional por corpo estranho superficial
}

\author{
Work-related eye trauma due to superficial foreign body
}

\author{
Fernando Antônio de Macedo Leal ${ }^{1}$ \\ Arthur Pereira da Silva e Filho ${ }^{2}$ \\ Daniela Martins Neiva ${ }^{3}$ \\ Josilene Carvalho Soares Learth ${ }^{4}$ \\ Durwagner Barros da Silveira ${ }^{5}$
}

\section{RESUMO}

Objetivo: Estudar a ocorrência de trauma ocular provocado por corpos estranhos superficiais durante a realização de atividades ocupacionais. Métodos: Estudo prospectivo de pacientes portadores de trauma ocular ocupacional atendidos no serviço de urgência do Hospital Getúlio Vargas (HGV) / Universidade Federal do Piauí (UFPI), no período de outubro de 1997 a março de 1999. Os seguintes dados foram coletados: idade, sexo, profissão, olho afetado, atividade realizada durante o trauma, natureza do corpo estranho, localização do mesmo e doença ocorrida. Resultados: Foram estudados 713 pacientes, sendo 686 (96,21\%) do sexo masculino e $27(3,79 \%)$ do sexo feminino. Os pacientes foram acometidos principalmente na terceira década de vida, correspondendo a um total de 312 pacientes $(43,75 \%)$. Com relação à atividade desenvolvida durante o trauma, 158 pacientes $(22,16 \%)$ eram metalúrgicos, $153(21,46 \%)$ serralheiros e 126 $(17,67 \%)$ mecânicos. No tocante ao corpo estranho, $489(68,58 \%)$ eram ferro e $47(6,59 \%)$ outros metais. Referente a localização, 587 corpos estranhos $(81,64 \%)$ estavam localizados na córnea, $75(10,43 \%)$ na conjuntiva tarsal e 49 $(6,81 \%)$ na conjuntiva bulbar. Além dos corpos estranhos $361(48,39 \%)$ dos pacientes apresentaram abrasão córneo-conjuntival. Conclusão: $O$ corpo estranho superficial é importante causa de trauma ocular ocupacional. A atividade desenvolvida principalmente por indivíduos do sexo masculino na metalurgia, serralheria e mecânica foi a principal causa do trauma, sendo o ferro e outros metais os mais frequientes corpos estranhos encontrados.

Descritores: Acidentes de trabalho; Corpos estranhos no olho; Traumatismos oculares/ epidemiologia; Equipamentos de proteção; Córnea/lesões; Cegueira/prevenção e controle

\section{INTRODUÇÃ̃O}

Os acidentes oculares são bastante comuns, acarretando custos de ordem social, psicológica e econômica ${ }^{(1-2)}$. Estima-se que nos Estados Unidos ocorram aproximadamente 2,4 milhões de traumas oculares por ano. Destes, em torno de 1 milhão, são decorrentes de acidentes no ambiente de trabalho, sendo que $90 \%$ são leves e preveníveis com utilização de medidas simples de proteção $^{(2-4)}$. Na Inglaterra tais acidentes ocupam uma taxa de $45 \%$ a $52 \%$ dos traumas, sendo que, também $90 \%$ são considerados preveníveis ${ }^{(5)}$. No Brasil estudos relatam que $10 \%$ dos acidentes ocupacionais são oculares ${ }^{(1)}$.

Estudos mostram que a maioria dos acidentes oculares ocupacionais é superficial, correspondendo a $72 \%$ do total ${ }^{(6)}$. Estudos também relatam que os corpos estranhos são responsáveis pela maioria destes acidentes e por uma incidência que varia de $54,6 \%$ a $81,8 \%$ do total de traumas oculares ${ }^{(1-2,5,7-8)}$. De acordo com Kuhn et $\mathrm{al}^{(9)}$ o trauma ocular superficial é a injúria contusa do globo ocular resultante de um projétil, na qual o corpo estranho torna-se 
alojado na superfície ocular, sem ter provocado perfuração ocular.

No Brasil os estudos a respeito deste assunto são escassos. Os dados existentes dizem respeito principalmente aos traumas graves, que necessitam de admissão hospitalar e que provocam muitos dias de afastamento do trabalho. Neste contexto, o objetivo deste estudo é avaliar a ocorrência de trauma provocado por corpos estranhos superficiais e ocorridos durante a realização de atividades profissionais, fornecendo subsídios para a adoção de medidas preventivas e educacionais adequadas.

\section{MÉTODOS}

Foram avaliados, prospectivamente, todos os pacientes portadores de trauma provocado por corpos estranhos superficiais e ocorridos em conseqüência da realização de atividades profissionais, que procuraram o serviço de urgência do pronto socorro do Hospital Getúlio Vargas (HGV) / Universidade Federal do Piauí (UFPI), no período de outubro de 1997 a março de 1999. Todos os atendimentos foram realizados por médicos e residentes do serviço de oftalmologia, sendo preenchido um formulário padrão no qual foram coletados os seguintes dados: idade, sexo, profissão, procedência, olho afetado, atividade realizada durante o trauma, natureza do corpo estranho, localização do mesmo e doença ocorrida.

\section{RESULTADOS}

Durante o período foram estudados 713 pacientes, sendo $686(96,21 \%)$ pacientes do sexo masculino e $27(3,79 \%)$ do sexo feminino. A maioria dos pacientes foi acometida principalmente na terceira década de vida, correspondendo a um total de $312(43,75 \%)$ pacientes (Gráfico 1). O olho esquerdo foi acometido em 55\% dos casos, o direito em 42,5\%. Em 2,5\% do total, ambos os olhos foram acometidos.

Do total de pacientes, $158(22,16 \%)$ foram vítimas do trauma durante a realização de atividades metalúrgicas, 153

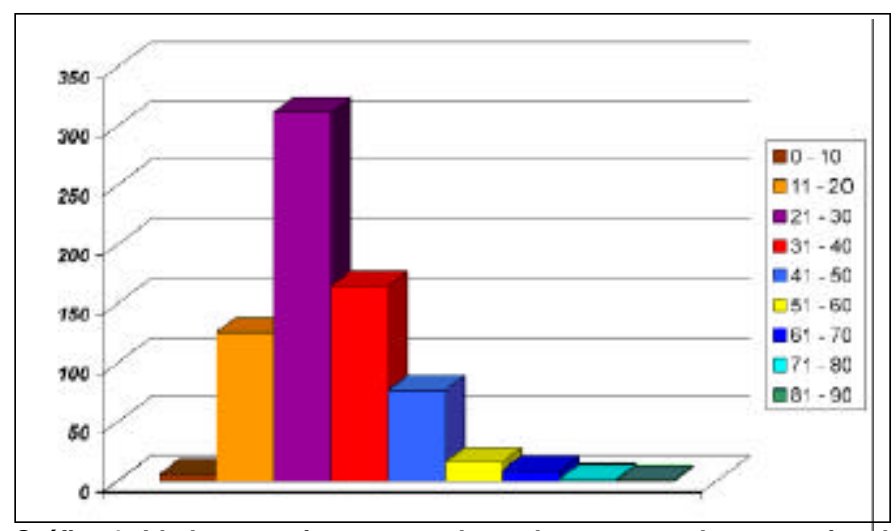

Gráfico 1 - Idade em pacientes portadores de trauma ocular ocupacional por corpos estranhos superficiais
$(21,46 \%)$ durante a serralheria, $126(17,67 \%)$ realizavam trabalhos mecânicos, $82(11,50 \%)$ traumas ocorreram na construção civil (pedreiro, marceneiro, carpinteiro, servente da construção civil e eletricista), 81 (11,36\%) estavam prestando serviços gerais (porteiros, caseiros, copeiros, motoristas, etc) e 56 $(7,85 \%)$ pacientes prestavam serviços agrícolas (Tabela 1 ).

Com relação aos corpos estranhos, $489(68,58 \%)$ eram ferro, $47(6,59 \%)$ outros metais e $81(11,36 \%)$ eram madeira (Tabela 2$)$. Referente à localização, $587(81,64 \%)$ dos corpos estranhos foram encontrados na córnea, $75(10,43 \%)$ na conjuntiva tarsal, $49(6,81 \%)$ na conjuntiva bulbar (Tabela 3$)$. Com relação à doença ocorrida, $361(48,39 \%)$ apresentaram abrasão corneana e/ou conjuntival, $217(29,08 \%)$ inflamação e $30(4,02 \%)$ úlcera corneana (Tabela 4).

\section{DISCUSSÃO}

Durante o período de 18 meses foram atendidos 713 pacientes vítimas de trauma ocupacional provocados por corpos estranhos superficiais. O termo trauma ocupacional é abrangente, incluindo trabalhadores formais e informais, descartando atividades que não fossem habitualmente desenvolvidas pelos pacientes. Do total destes, $96,21 \%$ eram do sexo masculino e $3,79 \%$ do sexo feminino. O dado é próximo dos $96 \%$ encontrados no México ${ }^{(8)}$, dos $98,65 \%$ encontrados no estado do Alabama (EUA) ${ }^{(10)}$ e dos $98,8 \%$ encontrados em Glasgow (Escócia) ${ }^{(5)}$. No Brasil estudos relatam incidências variando de $88,1 \%$ a $87 \%$ de traumas oculares ocupacionais no sexo masculino ${ }^{(1-2)}$. A maior incidência do trauma ocupacional em homens é amplamente relatada na literatura mundial, sendo atribuída ao fato destes exercerem maior atividade ${ }^{(1-3)}$ e serem menos cuidado$\operatorname{sos}^{(1)}$. Somente $3,79 \%$ dos acidentes ocorreram em pacientes do sexo feminino, de acordo com a literatura, que relata baixa incidência de acidentes oculares ocupacionais em mulheres ${ }^{(2,11)}$.

O trauma ocorreu principalmente na faixa etária de 20 a 30 anos, correspondendo a um total de 312 pacientes $(43,75 \%)$.

\begin{tabular}{|lrr|}
\hline \multicolumn{3}{|c|}{$\begin{array}{c}\text { Tabela 1. Trauma por corpos estranhos superficiais de acordo } \\
\text { com a atividade exercida }\end{array}$} \\
Atividade & No de Pacientes & $\%$ \\
Metalúrgico & 158 & 22,16 \\
Serralheiro & 153 & 21,46 \\
Mecânico & 126 & 17,67 \\
Prestação de serviços & 81 & 11,36 \\
Agrícola & 56 & 7,85 \\
Pedreiro & 31 & 4,34 \\
Marceneiro & 15 & 2,10 \\
Carpinteiro & 13 & 1,82 \\
Servente construção civil & 12 & 1,68 \\
Eletricista & 11 & 1,54 \\
Montador industrial & 8 & 1,12 \\
Comerciante & 5 & 0,70 \\
Outros & 44 & 6,17 \\
Total & 713 & 100,00 \\
\hline
\end{tabular}




\begin{tabular}{|lcr|}
\hline \multicolumn{2}{|c|}{$\begin{array}{c}\text { Tabela 2. Natureza dos corpos estranhos superficiais em } \\
\text { pacientes com }\end{array}$} \\
$\begin{array}{l}\text { Natureza do } \\
\text { corpo estranho }\end{array}$ & $\begin{array}{r}\text { No de } \\
\text { pacientes }\end{array}$ & $\%$ \\
Ferro & 489 & 68,58 \\
Madeira & 81 & 11,36 \\
Outros metais & 47 & 6,59 \\
Areia & 23 & 3,22 \\
Pólvora & 11 & 1,54 \\
Aço & 9 & 1,26 \\
Vidro & 9 & 1,26 \\
Cerâmica & 8 & 1,12 \\
Produtos químicos & 7 & 0,98 \\
Outros & 29 & 4,06 \\
Total & 713 & 100,00 \\
\hline
\end{tabular}

\begin{tabular}{|lcr|}
\hline \multicolumn{2}{|c|}{$\begin{array}{c}\text { Tabela 3. Localização do corpo estranho superficial em } \\
\text { pacientes com }\end{array}$} \\
$\begin{array}{l}\text { Localização do } \\
\text { corpo estranho }\end{array}$ & $\begin{array}{r}\text { No de } \\
\text { pacientes }\end{array}$ & $\%$ \\
Córnea & 587 & 81,64 \\
Conjuntiva tarsal & 75 & 10,43 \\
Conjuntiva bulbar & 49 & 6,81 \\
Pálpebras & 4 & 0,55 \\
Pontos lacrimais & 2 & 0,27 \\
Episclera & 2 & 0,27 \\
Total & 719 & 100,00 \\
\hline
\end{tabular}

Estudos relatam que o trauma ocupacional ocorre principalmente entre os 20 e $40 \operatorname{anos}^{(1-2,7,11)}$. Neste trabalho a incidência na faixa etária dos 11 a 20 anos $(17,62 \%)$ é semelhante à incidência na faixa etária dos 31 a 40 anos $(17,48 \%)$. A tendência a um aumento da incidência do trauma ocular em pacientes jovens no Brasil já é apontada por Kara-José Junior et al. ${ }^{(1)} \mathrm{e}$ Andrade et al. ${ }^{(2)}$. Aguilar ${ }^{(8)}$ enfatiza que os mais jovens se cuidam menos, chegando mesmo a desprezar os equipamentos de segurança. Com relação à lateralidade, não louve diferença significativa entre os olhos acometidos, conforme relatado em outras pesquisas.

No tocante à atividade profissional, 22,16\% dos traumas ocorreram durante atividade metalúrgica. Esta atividade foi responsável por $29,9 \%$ traumas oculares por acidentes de trabalho em um estudo realizado na cidade de Manaus entre fevereiro de 1991 a dezembro de $1997^{(7)}$. A serralheria foi responsável por $21,46 \%$ dos acidentes e a mecânica por $17,67 \%$, segundo este mesmo trabalho estas atividades tiveram incidências de $51 \%$ e $10 \%$ respectivamente ${ }^{(7)}$. Os trabalhos desenvolvidos na construção civil e prestação de serviços agrícolas foram responsáveis por $11,50 \%$ e 7,85\% dos traumas, respectivamente. $\mathrm{O}$ dado é semelhante ao relatado na literatura brasileira em dados coletados no período de 1991 a $1992^{(7)}$, mas difere dos dados coletados em uma pesquisa sobre acidentes oculares graves decorrentes do trabalho nos anos de 1977 a $1991^{(11)}$. Nesta pesquisa, a agricultura e a construção civil

\begin{tabular}{|c|c|c|}
\hline Doença ocorrida & № de pacientes & $\%$ \\
\hline $\begin{array}{l}\text { Abrasão de córnea } \\
\text { e/ou conjuntiva }\end{array}$ & 361 & 48,39 \\
\hline Inflamação & 217 & 29,08 \\
\hline Úlcera & 30 & 4,02 \\
\hline Infecção & 14 & 1,87 \\
\hline Hemorragia & 12 & 1,61 \\
\hline Nenhuma & 112 & 15,01 \\
\hline Total & 746 & 100,00 \\
\hline
\end{tabular}

foram as principais atividades responsáveis pelos acidentes, com incidências de $22 \%$ e $30 \%$ respectivamente. Do total de traumas, 11,36\% ocorreram com prestadores de serviços gerais tais como: motoristas, copeiros, zeladores, etc.

De acordo com a literatura os traumas oculares são em grande parte superficiais e/ou provocados por corpos estranhos ${ }^{(1-7)}$. Em um trabalho específico sobre corpos estranhos de córnea, 91,58\% dos casos foram decorrentes de acidentes de trabalho ${ }^{(2)}$. Neste estudo, dos corpos estranhos provocadores do trauma ocupacional, $75,17 \%$ eram de natureza metálica. Em um estudo sobre acidentes oculares ocupacionais as maiores incidências foram na indústria da construção civil e na indústria do metal, sobretudo o ferro. Com relação à localização, os corpos estranhos foram encontrados principalmente na córnea e conjuntiva, correspondendo a um total de $98,88 \%$ dos casos. A literatura relata incidência de 54,6\% de corpo estranhos córneoconjuntivais dentre traumas oculopalpebrais ${ }^{(2)}$. Além dos corpos estranhos as principais doenças ocorridas foram abrasão da córnea e/ou conjuntiva, 48,39\%, e inflamação, 29,08\%.

Neste trabalho preferimos usar o termo corpo estranho superficial ao invés de corpo estranho extra-ocular, comumente usado em nossa literatura, procurando adequar o termo ao sistema de classificação padronizado para os mecanismos de trauma do globo ocular, proposta por Kunh et al. ${ }^{(9)} \mathrm{e}$ aceito atualmente pela International Society of Ocular Trauma, United States Injury Registry, Vitreous and Retinal Society e American Academy of Ophthalmology $y^{(1,3)}$.

Dois importantes fatores quando se estuda o trauma ocupacional não foram aferidos neste estudo: o número de dias de ausência no trabalho e o uso de proteção. Com relação ao primeiro, Kara-José Junior et al. ${ }^{(1)}$ relatam em média de 4,8 dias de afastamento do trabalho em um estudo sobre acidentes oculares ocupacionais ocorridos em Botucatu, no período de 1998 a1992. Andrade et al. ${ }^{(2)} \mathrm{em}$ um estudo realizado em 1988 no Hospital Universitário Evangélico de Curitiba relatam que houve uma necessidade de 3 a 5 dias, em média, para a cura de 95,5\% dos pacientes com lesões oculares leves do tipo abrasão corneana. Em relação ao uso de proteção, a literatura mundial relata amplamente que a maioria dos traumas ocorre na ausência de seu uso ${ }^{(3,5-7,9)}$, mesmo quando esta estava disponível ${ }^{(5-6)}$ e que os traumas superficiais são facilmente preveníveis se a proteção for usada adequadamente ${ }^{(12)}$. 


\section{CONCLUSÃO}

Os corpos estranhos superficiais, facilmente preveníveis com o uso de proteção adequada, constituem uma importante causa de traumas ocupacionais, trazendo perdas econômicas, sociais e psicológicas. Neste estudo a atividade desenvolvida principalmente por homens na metalurgia, serralheria e mecânica foi a principal causa de trauma ocular, e o ferro e outros metais os principais corpos estranhos envolvidos.

\section{ABSTRACT}

Purpose: To study the prevalence of superficial ocular trauma due to foreign bodies in patients during occupational activities. Methods: Prospective study of patients with occupational ocular trauma diagnosed at the Hospital Getúlio Vargas (HGV) / Universidade Federal do Piauí (UFPI), from October 1997 to March 1999. The following data were obtained: age, gender, occupation, affected eye, activity performed at the time of the trauma, nature and localization of the foreign body and posterior complications. Results: 713 patients were studied, of whom $686(96.21 \%)$ were males and $27(3.79 \%)$ were females. The patients were affected mainly in the third decade of life, corresponding to 312 patients $(43.75 \%)$ ). In relation to the activity performed during the trauma, 158 patients (22.16\%) were metallurgists, $153(21.46 \%)$ locksmiths and 126 $(17.67 \%)$ mechanics. Concerning the foreign body, 489 $(68.58 \%)$ were iron and $47(6.59 \%)$ were other metals. Regarding localization, 587 foreign bodies $(81.64 \%)$ were in the cornea, $75(10.43 \%)$ in the tarsal conjunctiva, $49(6.81 \%)$ in the bulbar conjunctiva. In addition to the foreign bodies 361 $(48.38 \%)$ patients presented corneo-conjunctival abrasion.
Conclusion: The superficial foreign body is an important cause of occupational ocular trauma. The most frequent component was iron, and the patients were mainly metallurgists, locksmiths and mechanics, males in their thirties.

Keywords: Occupational accidents; Eye foreign bodies; Eye injuries/epidemiology; Protective devices; Cornea/injuries; Blindness/prevention and control

\section{REFERÊNCIAS}

1. Kara-José Jr N, Oliveira-Neto JC, Silva ALB, Schellini SA. Acidentes oculares ocupacionais - ocorrência em Botucatu, no período de 1988 a 1992. Arq Bras Oftalmol 1994;57:389-93.

2. Andrade AS, Bisneto OS, Moreira H, Moreira CA. Traumas oculo-palpebrais no Serviço de Pronto-Atendimento Oftalmológico do Hospital Universitário Evangélico de Curitiba. Arq Bras Oftalmol 1999;62:585-9.

3. Hall E. Protective eyewear, proper care help stop injuries, blindness at work. Occup Health Saf 1987;56:70-1,80.

4. Rosenwasser TL, Potter JW, Parr RB. Vision losses prevented by using protective eyewear. Occup Health Saf 1985;54:63-6.

5. Macewen CJ. Eye injuries: a prospective survey of 5671 cases. Br J Ophthalmol 1989;73:888-94.

6. Saari KM, Parvi V. Occupational eye injuries in Finland. Acta Ophthalmol 1984;161(Suppl):17-28.

7. Cohen J, Carvalho RC, Romão E. Trauma ocular por acidente de trabalho em Manaus. Rev Bras Oftalmol 1994;53:69-72.

8. Aguilar F. Repercussiones sócio-econômicas de las lesiones por cuerpos extraños. Salud Publica México 1985;27:66-70.

9. Kuhn F, Morris R, Witherspoon CD, Heimann K, Jeffers JB, Treister G. A standized classification of ocular trauma[commented on Ophthalmology 1997;104:344-5]. Ophthalmology 1996;103:240-3.

10. White MF, Morris R, Feist RM, Witherspoon CD, Helms HA, John GR. Eye injury: prevalence and prognosis by setting. South Med J 1989;82:151-8.

11. Schellini SA, Marchi NLM, Itoda LK, Silva MRBM, Sab N. Acidentes oculares graves decorrentes do trabalho. Rev Bras Oftalmol 1993;52:55-62.

12. Spada FR, Rodrigues EB, Grumann Jr A, Cunha ETSR. Corpo estranho de córnea: relação com a atividade profissional. Rev Bras Oftalmol 2000;59:36-9. 